\title{
Plasmonic metadevices by vertical split ring resonator
}

Wei-Yi Tsai ${ }^{1}$, Mu-Ku Chen ${ }^{l}$, Pei Ru Wu ${ }^{1}$,Yi-Hao Chen ${ }^{1}$, Ting-Yu Chen ${ }^{1}$, Jia-Wern Chen ${ }^{1}$, Cheng Hung Chu ${ }^{2}$, Pin Chieh $W^{2}$, Chun Yen Liao ${ }^{1}$, Huijun Wu ${ }^{2}$, Hsiang-Chu Wang ${ }^{2}$, Greg Sun ${ }^{3}$, Ai Qun Liu ${ }^{4}$, and Nikolay I. Zheludev ${ }^{5}$, Din Ping Tsai ${ }^{1,2 *}$

\author{
${ }^{1}$ Department of Physics, National Taiwan University, Taipei 10617, Taiwan \\ ${ }^{2}$ Research Center for Applied Sciences, Academia Sinica, Taipei 11529, Taiwan \\ ${ }^{3}$ Department of Physics, University of Massachusetts Boston, Boston, Massachusetts 02125, USA \\ ${ }^{4}$ School of Electrical and Electronic Engineering, Nanyang Technological University, Singapore 639798, Singapore \\ ${ }^{5}$ Optoelectronics Research Centre, University of Southampton, Southampton SO17 1BJ, UK \\ *corresponding author, E-mail: dptsai@phys.ntu.edu.tw
}

Split-ring resonator (SRR), one kind of building block of metamaterials, has attracted wide attentions due to the resonance excitation of electric and magnetic dipolar response. Fundamental plasmon properties and potential applications in novel three dimensional vertical split-ring resonators (VSRRs) are designed and investigated. The resonant properties arose from the electric and magnetic interactions between the VSRR and light are theoretically and experimentally studied. Tuning the configuration of VSRR unit cells is able to generate various novel coupling phenomena in VSRRs, such as plasmon hybridization and Fano resonance. The magnetic resonance plays a key role in plasmon coupling in VSRRs. The VSRR-based refractive-index sensor will be demonstrated, as shown in Figs. 1. Due to the unique structural configuration, the enhanced plasmon fields localized in VSRR gaps can be lifted off from the dielectric substrate, allowing for the increase of sensing volume and enhancing the sensitivity. We perform a VSRR based metasurface for light manipulation in optical communication frequency, as shown in Fig. 1 as well. By varying the prong heights, the $2 \pi$ phase modulation can be achieved in VSRR for the design of metasurface. Because the phase shift is changed via the upright configuration rather than the one parallel to the substrate, it can be used for high areal density integration of metal nanostructures and opto-electronic devices. Fabrication of three dimensional VSRR by stress-driven assembly method for uniaxial-isotropic metamaterials is demonstrated as well.

(a)

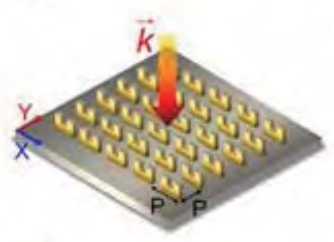

(e)

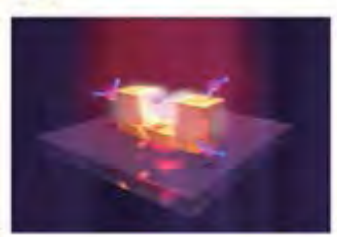

(b)

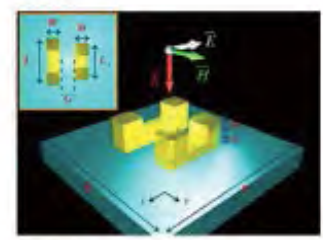

(f)

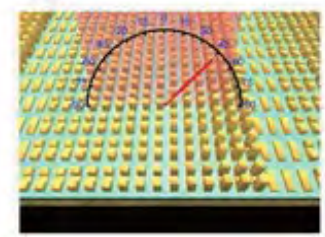

(c)

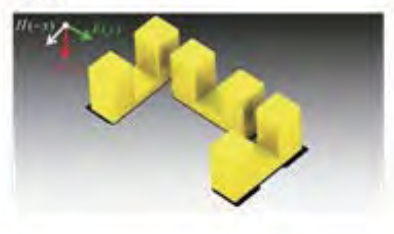

(g)

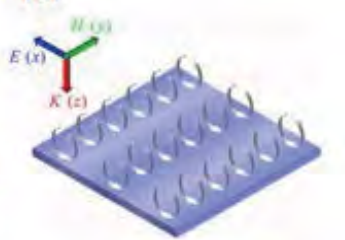

(d)

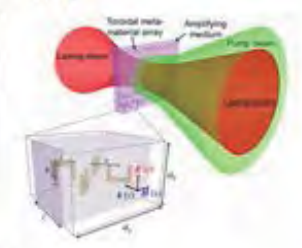

(h)

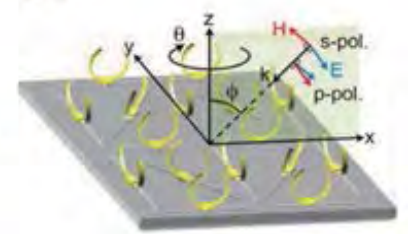

Figure 1. Schematic diagrams for (a) VSRR (b) VSRR based plasmon coupling, (c) Fano resonance, (d) toroidal metamaterial, (e) nanoplasmonic sensor, (f) metasurface, (g) tunable VSRR and (h) uniaxial-isotropic metamaterials.

\section{References:}

1. P. C. Wu, W.-L. Hsu, W. T. Chen, Y.-W. Huang, C. Y. Liao, A. Q. Liu, N. I. Zheludev, G. Sun, and D. P. Tsai, Sci. Rep. 5, 9726, 2015.

2. C.-C. Chen, A. Ishikawa, Y.-H. Tang, M.-H. Shiao, D. P. Tsai, T. Tanaka, Adv. Opt. Mater. 3, 44-48, 2015. 